\title{
A DIGITAL SYSTEM FOR ACCURATE TIME SECTOR DIVISION OF A SPIN STABILIZED VEHICLE
}

\author{
by \\ W. C. B artley \\ X.6. Hacracken* \\ U.R. Rao**
}

This work was initially supported by NASA Contract NASr-198, while the construction of the flight hardware was supported by contract NAS2 -1756 . Data analysis is Derformed under NASA contract NSR-44-004-043

* Now at University of Adelaide, Adelaide, South Australla, Australla

* *Now at Physical Research Laboratories, Ahmedabad, India.

SOUTHWEST CENTER FOR ADVANCED STUDIES P. $0.80 x 30365$, Dallas, Toxas $\mathbf{7 5 2 3 0}$

Division of

GRADUATE RESEARCH GEMTER OF THE SOUTHWEST
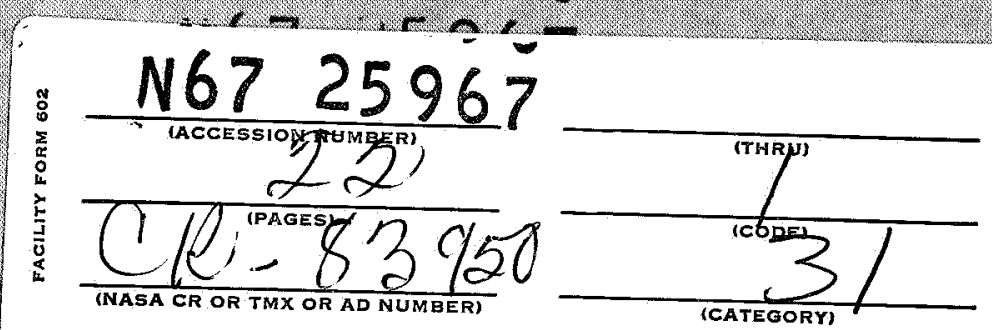

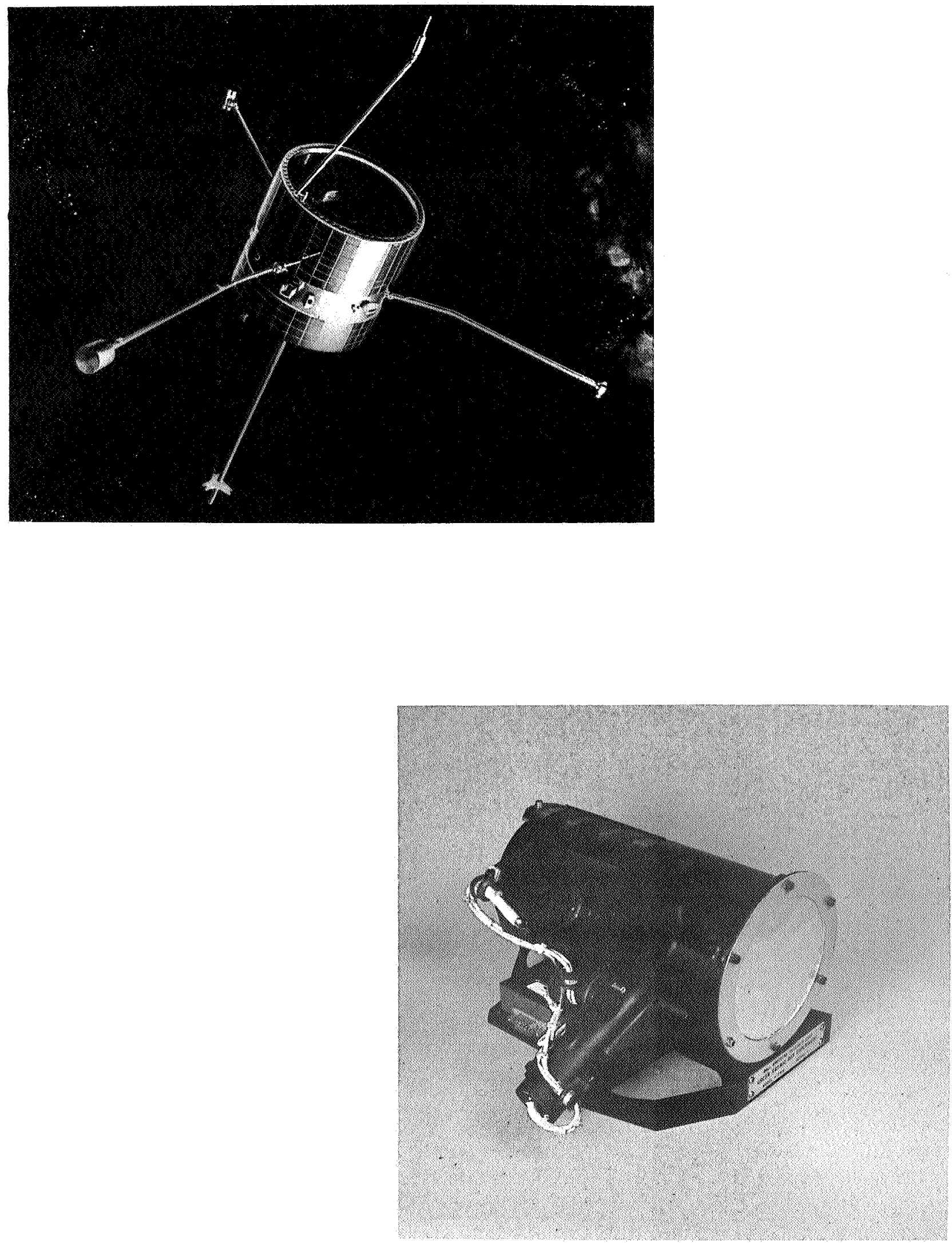


\section{A DIGITAL SYSTEM FOR ACCURATE TIME SECTOR \\ DIVISION OF A SPIN STABLIZED VEHICLE}

by

\section{W. C. Bartley, K. G. McCracken*, and U. R. Rao** Southwest Center for Advanced Studies}

Dallas, Texas

\section{INTRODUCTION}

In studying the physical properties of the interplanetary medium, the manner in which the various parameters describing the medium depend upon direction is of major theoretical importance. For example: the angular dependence of plasma properties, the distribution in direction of meteoroid arrival vectors, and the angular dependence of the cosmic radiation flux, to name a few, are important relationships which give great insight into the physics of the solar system. For this reason, a number of contemporary spacecraft have been designed with such directional studies as major objectives and there will be increasing emphasis on such studies in the future. When all the relevant factors are taken into account, it is found that a spacecraft spinning about a single axis fixed in inertial space is the best vehicle for such studies, as exemplified by satellites of the IMP or the Pioneer classes. In such a satellite, the determination of direction of viewing can be transformed into the measurement of time elapsed since the detector pointed in some known direction (which is most commonly the sun) .

* Now at University of Adelaide, Adelaide, South Australia, Australia

** Now at Physical Research Laboratory, Ahmedabad, India 
In measuring some physical phenomena as functions of spacecraft rotation azimuth, it is often true that the rate at which the phenomena change is slow compared to the spin period of the satellite. Consequently, transmission of the measured quantity as a function of azimuth on each and every spin rotation of the satellite is extremely wasteful of spacecraft telemetry capacity, and some on-board averaging prior to data transmission results in a great saving in the bandwidth required, without adversely affecting the usefulness of the resulting data. That is, on-board data computation can result in considerable conservation of telemetry bandwidth, at the expense of added complexity on the spacecraft. In many modern satellites, and deep space probes, this is the preferable compromise. In this paper, we describe a system flown on the Pioneer VI and VII spacecrafts, and to be flown on other vehicles, which contains a highly accurate digital directional computer, and which, in addition, incorporates extremely simple data computation to minimise telemetry bandwidth requirements. The in-flight performance of the complete system is reviewed: in particular, the continuous adaption to changes in the spacecraft spin frequency is demonstrated and the accumulation time accuracy (vide infra) is shown to be better than 2.5 parts in $10^{5}$ at all times during more than nine months of continuous in-flight operation.

\section{SYSTEM CONSTRAINTS}

The experiments to be described were designed to measure the cosmic radiation flux as a function of direction.

\section{[1] A simplified block} diagram (Figure 1) illustrates the operation of the Pioneer VI and VII experiments and will permit definition of the most important system constraint. Similar considerations apply to the other spacecraft systems. A directional cosmic ray detector is mounted on the spacecraft, such as to record cosmic rays arriving from directions normal to the spacecraft spin 


\section{$-3-$}

axis. The complete $360^{\circ}$ of spacecraft spin rotation is divided up into four "Quadrants" of about $89.5^{\circ}$ each (se Figure 2). Corresponding to each output of the pulse height analyser in the detector, there are four binary accumulators, one for each of the four quadrants of satellite spin rotation. Each time a pulse appears at the pulse height analysis output, it is routed to the appropriate quadrant accumulator via the gate which is, at the instant, enabled. This process is repeated for a number of spin periods, after which time the data within the accumulator are read out to the spacecraft telemetry encoder. In the manner, the cosmic ray counting rate is computed as a function of spacecraft azimuth.

The major system constraint is that an anisotropy (i,e, , a directional dependence) of the cosmic radiation as small as 1 part in $10^{3}$ has to be capable of detection without ambiguity $[2,3,4]$ This implies that the accumulation times for the 4 quadrant accumulators must exhibit an equality of this precision or better, in order that differences in accumulation time should not produce results which would be taken to imply an anisotropy in the cosmic radiation. To be conservative, a design goal of equality of accumulation time to within 1 part in $10^{4}$ was set. Since the spin frequency of a spacecraft changes with time, an adaptive system is necessary.

The digital "Aspect clock" designed to meet the above design goal has consistently surpassed the above design goals during the six months of flight of Pioneer VI. The various systems of a similar nature developed in this laboratory divide the spin period of the vehicle into either 32, 8 or 4 rigorously equal intervals of time and consequently in what follows, we describe a generalize $2^{\mathrm{m}}$ interval aspect clock. 


\section{GENERAL THEORY OF OPERATION}

The basic logic of the aspect clock is presented in (Figure 3). A suitable frequency source $£$ feeds two cascaded ripple-through counters, COUNTER-M and COUNTER-N, via a gate which is controlled by a suitably shaped pulse generated by an optical aspect sensor located on the perimeter of the spinning spacecraft. COUNTER-M consists of M binaries, hence the output frequency is $f / 2^{m}$. In the period of time $\mathrm{T}$ between the cessation of one sun pulse, and the commencement of the next, $N$ pulses are counted by COUNTER-N, where $\mathrm{N}$ is given by $\mathrm{Tf}=2 \mathrm{~m} N+\mathrm{R}$, where $\mathrm{R}$, the remainder, is less than $2^{\mathrm{m}}$. That is, $\mathrm{N}$ is an adaptive measure of the spin period of the spacecraft.

At the instant at which the optical aspect pulse closes the input gate to Counter-M, the content of Counter-N is parallel shifted to REGISTER-N, where it acts as a spin period reference during the subsequent spin period. Each corresponding bit of COUNTER-N' and REGISTER-N feedsinto a parallelcomparison-gate (in the form of a "half adder" or an "exclusive OR" depending upon the logic form used). The outputs of these comparison gates feed into an n-input "AND" gate. Hence, each time that all corresponding bits in REGISTER-N and COUNTER-N' are at the same logical state, a coincidence pulse is produced at the "AND" gate output, and Counter $-N^{\prime}$ is reset to zero. Since the input pulse rate to Counter $-N^{\prime}$ is $f$, it will repeat this cycle $2^{\mathrm{m}}$ times during the period of time T, and will contain a remainder $\mathrm{R}$ at the commencement of the subsequent sun pulse.

The $2^{\mathrm{m}}$ coincidence pulses which are generated during each spin period are separated from one another by exactly N/f seconds, and hence the pulse separation equality is limited only by the short term stability of $f$. In practice, $f$ is crystal controlled, and hence the intervals of time defined by the coincidence pulses are equal to within about 1 part in $10^{5}$ or better, 
Since the spin rates of most spacecraft change over a period of time due to torques introduced by light pressures on the solar panels, solar wind pressures, or out-gassing from orientation systems, a system such as this must be capable of accomnodating to gradual changes in spin period. If the remainder $\mathrm{R}$ were to be zero in the system just described the next sun pulse might occur before the final $2^{\mathrm{m}}$-th coincidence pulse from the "AND" gate has occurred, For example, such a "race" problem would occur when $\mathrm{R}=\mathrm{O}$ due to the asynchronous nature of the spacecraft sun period, and the frequency f. Likewise, if the spacecraft were to be precessing, the time interval between successive sun pulses would show a sinusoidal variation with time, which would introduce rate problems. These problems can be easily eliminated by introducing a small but deliberate dead time period prior to each sun acquisition. The implementation of such a dead time can be quite simple (for instance resetting COUNTER-N to all "ones" instead of "zeros" following a sun acquisition) and this dead time will do no harm to the scientific measurements provided that $\mathrm{N}$ is reasonably large $(\mathbb{N}>100)$,

In the Pioneer VI aspect clock $f=5.461 \mathrm{kc} / \mathrm{sec}, \mathrm{m}=5$, and the nominal spin period $\mathrm{T}=1.0$ second. To provide a fixed dead time of $5.86 \mathrm{msec}$, the M-Counter is reset to "all ones" during the sun pulse. Hence, the number N in the $\mathrm{N}$-Counter after one spin period $=172$. Since the remainder $\mathbf{R}$ can be as great as $\left(2^{\mathrm{m}}-1\right) / \mathrm{f}$, there is a variable dead time of maximum deviation $5.86 \mathrm{msec}$ and the total deadtime can lie within the range 5.86 to 11.72 milliseconds, corresponding to between 2 and 4 degrees of spacecraft rotation. At any given time during flight, the deadtime duration is known with an accuracy of better than $\pm 0.2 \mathrm{msec}$. In Figure $4 \mathrm{a}$ the temporal variation of the spacecraft spin period, and the dead time are displayed for four months of flight. 
The aspect clock is tolerant to wide deviations from the nominal spacecraft spin period and to variations in F. The limiting spin period will be such that the combined $\mathrm{M}$ and $\mathrm{N}$-Counters overflow during a single spin period: $-1, \theta$, the maximum spin period $T_{m}$ for the Pioneer VI system is given by $2^{13}=5.461 \times T_{m}, 1 . e . T_{m}=1.5 \mathrm{sec}$. If it were deemed desirable to extend the limits of operation, further binary stages could be added to the $\mathrm{N}$-Counter.

An inflight verification of the equality of time division is obtained by feeding a $6.7 \mathrm{kc} / \mathrm{sec}$ pulse rate to the quadrant gate inputs for one spin rotation during the calibration sequence which is initiated by radio command once every 4 hours. In this way it has been possible to demonstrate that the various quadrant accumulation times have at all times been equal to within 2.5 parts in $10^{5}$. Since the time interval between the successive 2 coincidence pulses is $N / f, i, e$, , it is quantised in units of $l / f$ seconds, the accumulation time in a system such as flown on Pioneer VI exhibits a discontinuous change each time $\mathrm{N}$ changes (see Figure 4b). On Pioneer VI and VII, the quadrant accumulation times were "quantised" in units of $1.465 \mathrm{msec}$, hence the number of $6.7 \mathrm{kc} / \mathrm{sec}$ pulses counted during the calibration sequence changed abruptly by $10 \pm 1$ each time the number $N$ changed (as a consequence of the changing spin period). In this manner the operation of the aspect clock could be monitored at all times during the flight.

Bearing the design requirements of equality of accumulation time in mind, it will be clear that in addition to generating rigorously equal gating signals, the data must be read out from the quadrant accumulators in a manner which is synchronous with the sun. That is, an unbuffered read out into a conventional telemetry encoder, in which the time of readout is determined by the encoder timing circuitry, alone, is unacceptable. In the systems developed within this laboratory, the data are readout from 
the quadrant accumulators into a 24 bit buffer a dead time shortly before the encoder interrogates the experiment. At the time the experiment is interrogated, the data are readout from this buffer. This insures that all of the quadrant measurements correspond to accumulation during an identical number of complete spin periods of the spacecraft.

IV. IMPLEMENTATION

The aspect clock as flown on Pioneer VI and VII is constructed from about 60 TI Series-51 type integrated circuits. It weighs about 40 grames, and consumes 150 milliwatts of regulated power (a photo of the total interograted circuits signal conditioner electronics as shown in Fig. 5).

In the Pioneer instrument, $m=5$, hence, there are 32 coincidence pulses $\left(2^{\mathrm{m}}\right.$ pulses) per spin period, each representing a $\pi / 16$ rotation of the spacecraft. Under normal circumstances these pulses are scaled in a 3-bit counter to yield 4 pulses per spin period, these being used to define the four "quadrants" employed in the spacecraft; $i, e$, , the quadrants are defined by the logic combination $\overline{8} . \overline{16}, 8 . \overline{16}, \overline{8} .16$, and 8.16 . To reduce the accumulation time of each "Quadrant" by a factor of 8 , and to change the mean direction of viewing of the instrument, (see shaded area of Figure 2) it is merely necessary to generate the logic $S=\overline{1} \cdot \overline{2} . \overline{4}$ from the three binaries in the prescaler, and then define the four quadrants by the logic combinations $\overline{8} . \overline{16} .5,8 . \overline{16} .5, \overline{8} .16 .5$, and 8.16.S. In the Pioneer instrument this change is affected by radio command from earth whenever the cosmic ray counting rates increase to the point where the bit quadrant accumulators tend to overflow, An equivalent extension in dynamic range by prescaling after quadrant division would have required 48 additionalbinaries, and 96 additional gates, as compared to the 6 binaries and about 10 gates required to implement the above. 
Figure 6 showing the actual detailed schematic of the aspect clock system as implemented on Pioneer VI and VII is included for information to designers. Space does not allow an analysis of this schematic here. However, a good portion of the circuit is self explanatory from the generalized aspect clock block diagram (Figure 3) and the instrument logic (Figure 1).

It will be noted that this system features circuitry which inhibits the sun pulse signal except for a limited window of arc. This is to avoid difficulties due to potential noise on the sun sensor line. Because of this feature additional ancillary circuitry is provided to preclude the possibility of the system "locking up" on some integral multiple of the actual spin period (eg. $4 \pi$ radians instead of $2 \pi$ radians) During each ground commanded calibrate sequence of the instrument, the aspect system "looks" for a full $360^{\circ}$ for a sun pulse via a signal to the "2 $\pi$ Search" command line (see lower right portions of Figure 6).

Also, in this system, a free-run mode was provided in case there was either intermittent, or permanent failure of the optical aspect device. Thus, if a sur pulse had not been detected within milliseconds after its expected occurence, the aspect clock generated a simulated sun pulse, and continues to aspect divide, always using the last value of $\mathrm{N}$ successfully computed from the sun. The Pioneer VI instrument has never been observed to be in free-run.

\section{CONCLUSIONS}

The scientific information obtained from this GRCSW cosmic ray experiment utilizing the aspect dividing system described above has already lead to the discovery of some important features of the interplanetary 
magnetic field. Preliminary conclusions based on data received from Pioneer VI are discussed in other publications. [5,6] Early data from Pioneer VII are now being analyzed.

\section{ACKNONTEDGEMENTS}

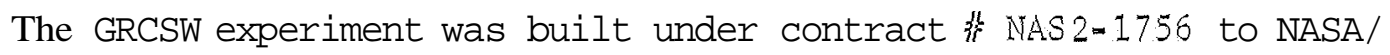
Ames Research Center. The aspect clock was constructed by Apparatus Division of Texas Instruments, Texas, with other portions constructed and integrated by Matrix Corporation, New Hampshire, and Ball Brothers Research Corporation, Colorado. Assistance of many kinds was provided by the Pioneer Project Manager, Mr. C. F. Hall and his staff. Many people contributed to the success of the experiment, however, particularly crucial contributions were made by H. Cross and C. Lewis (Ames); G. Crouse, J. Taylor and C. Prokesh (Texas Instruments); M. Poyer, G. Gillan, and V. Rogowski (ball Brothers); and Dr. R. P. Bukata, C. Melton and H. Glasscock (GRCSW) . 
REFERENCES

[1] W. C. Bartley, K. G. McCracken, and U. R. Rao, "The Pioneer VI Detector to Measure the Degree of Anisotropy of the Cosmic Radiation in the Energy Range 7.5-90 Mev/Nucleon" GRCSW Technical Report MDASS $=66-2,1966$.

[2] J. J. Quenby, Handbuch der Phys 1k, Bd 46, 2 (1964).

[3] U. R. Rao, K. G. McCracken and D. Venkatesan, Journal of Geophysical Research, Vol. 68, p 345, 1963.

[4] K. G. McCracken and U. R. Rao, Proceedings of the 9th International Conference on Cosmic Rays, IQSY Committee, London (1965),

[5] W. C. Bartley, R. P. Bukata, K。 G. McCracken, and U.R。Rao, "Anisotropic Cosmic Radiation Fluxes of Solar Orgin", Journal of Geophysical Research, Vol 71, p 3297, July 1966.

[6] K. G. McCracken and N. F. Ness, "The Collimation of Cosmic Rays by the Interplanetary Magnetic Field, Journal of Geophysical Research, Vol. 71, p 3305, July 1966. 
CAPTIONS

Figure 1 - Simplified Block Diagram of GRCSW Cosmic Ray Anisotropy Experiment on Pioneer VI and VII.

Figure 2 - Data gate signals timing as a function of the Pioneer Spacecraft Azimuth.

Figure 3 - Generalized "Aspect Clock" system logic.

Figure 4 - (a) Pioneer VI spin period as a function of time plotted with the corresponding aspect clock dead times.

(b) Quadrant accumulation time as measured by telemetered calibrate sequences plotted as a function of time showing discontinuities each time the number $\mathrm{N}$ changed.

Figure 5 - Photo shows complete signal conditioner package containing approximately 400 integrated circuits in welded modules mounted on two laminated six-layer printed circuit boards 3.0 inches in diameter. The "Aspect Clock" comprises approximately one-seventh of this package.

Figure 6 - Pioneer "Aspect Clock" schematic 


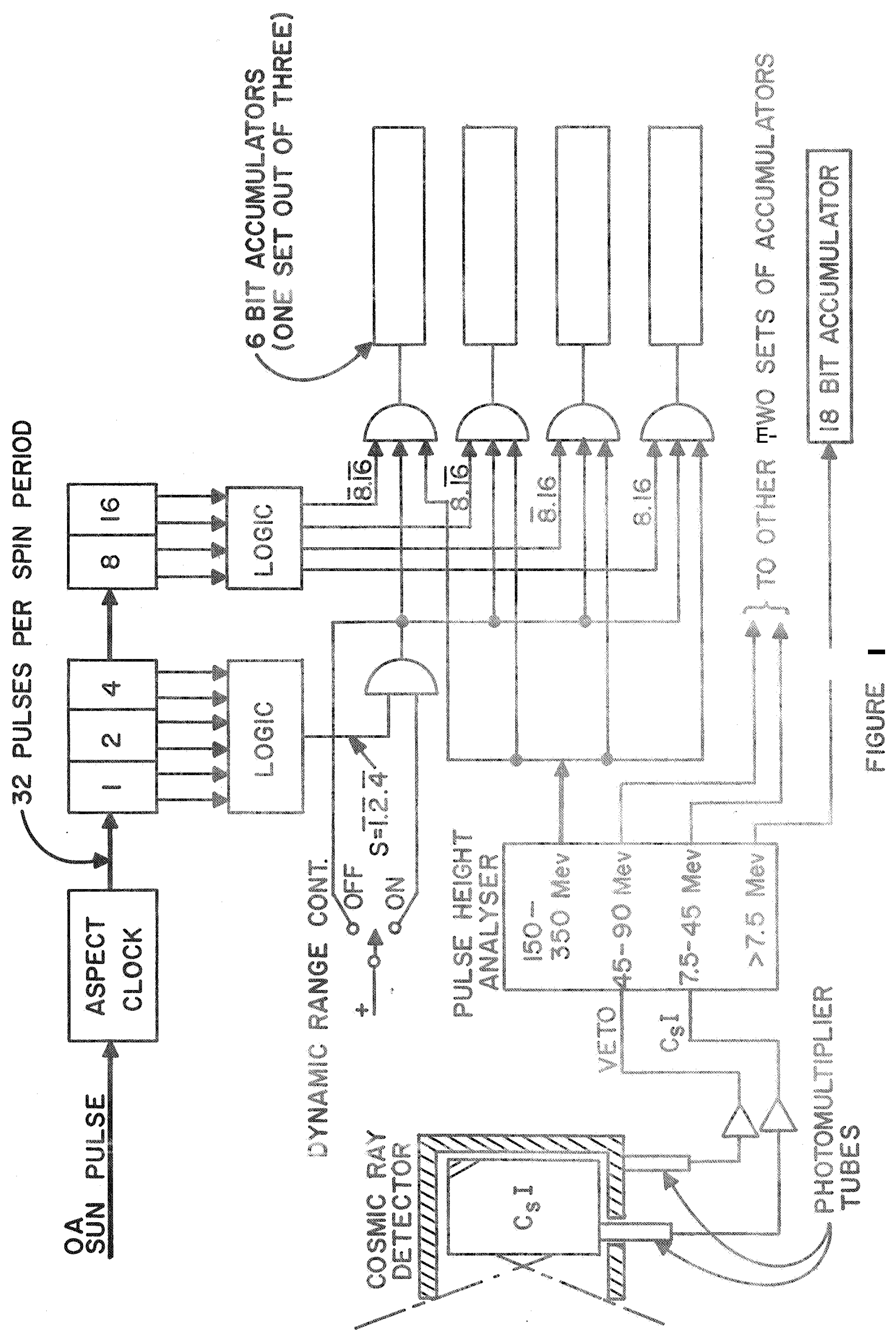


ASPECT CLOCK LOGIC

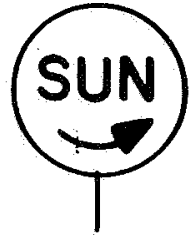
IN PARENTHESES

\section{PIONEER SENSE OF ROTATION}
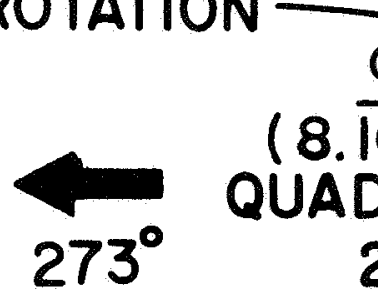

$325^{\circ}$
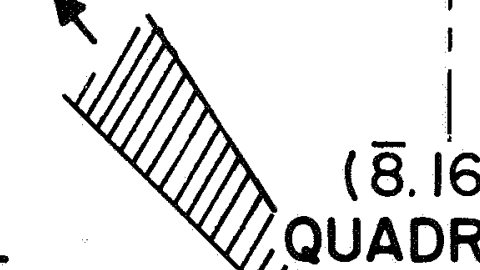

$(\overline{8} .16)$

QUADRANT

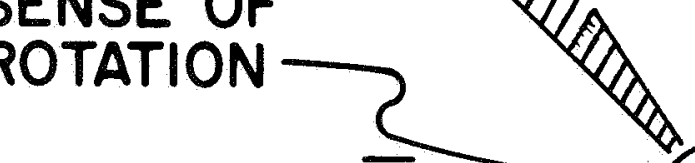

QUADRANT

2

$1 / \overline{8} \cdot \overline{16})$

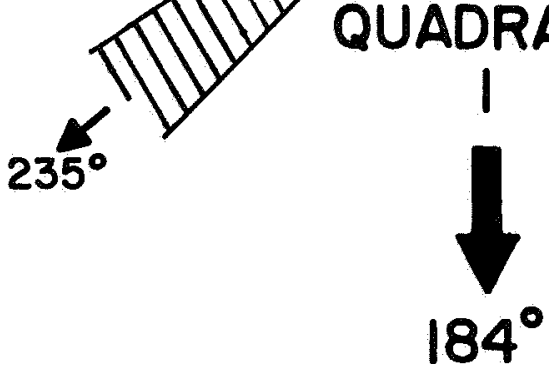

$003^{\circ} \quad(s=\bar{T} . \overline{2} . \overline{4})$

$055^{\circ}$
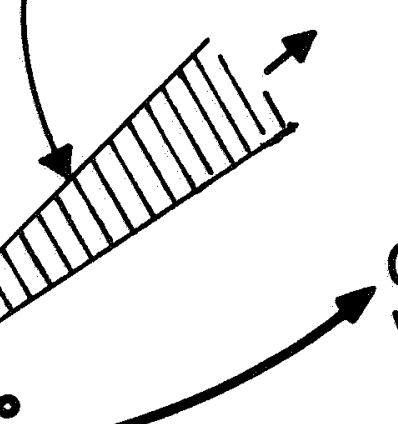

ORBITAL VELOCITY

(8.16)

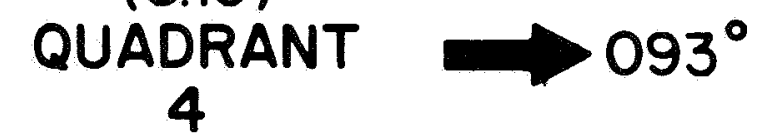
4

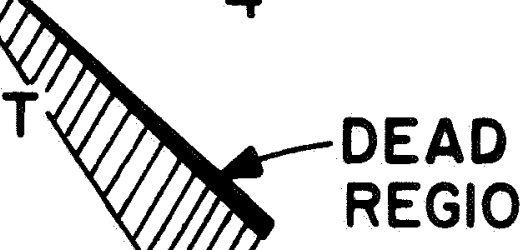

\section{REGION}

FIGURE 2 


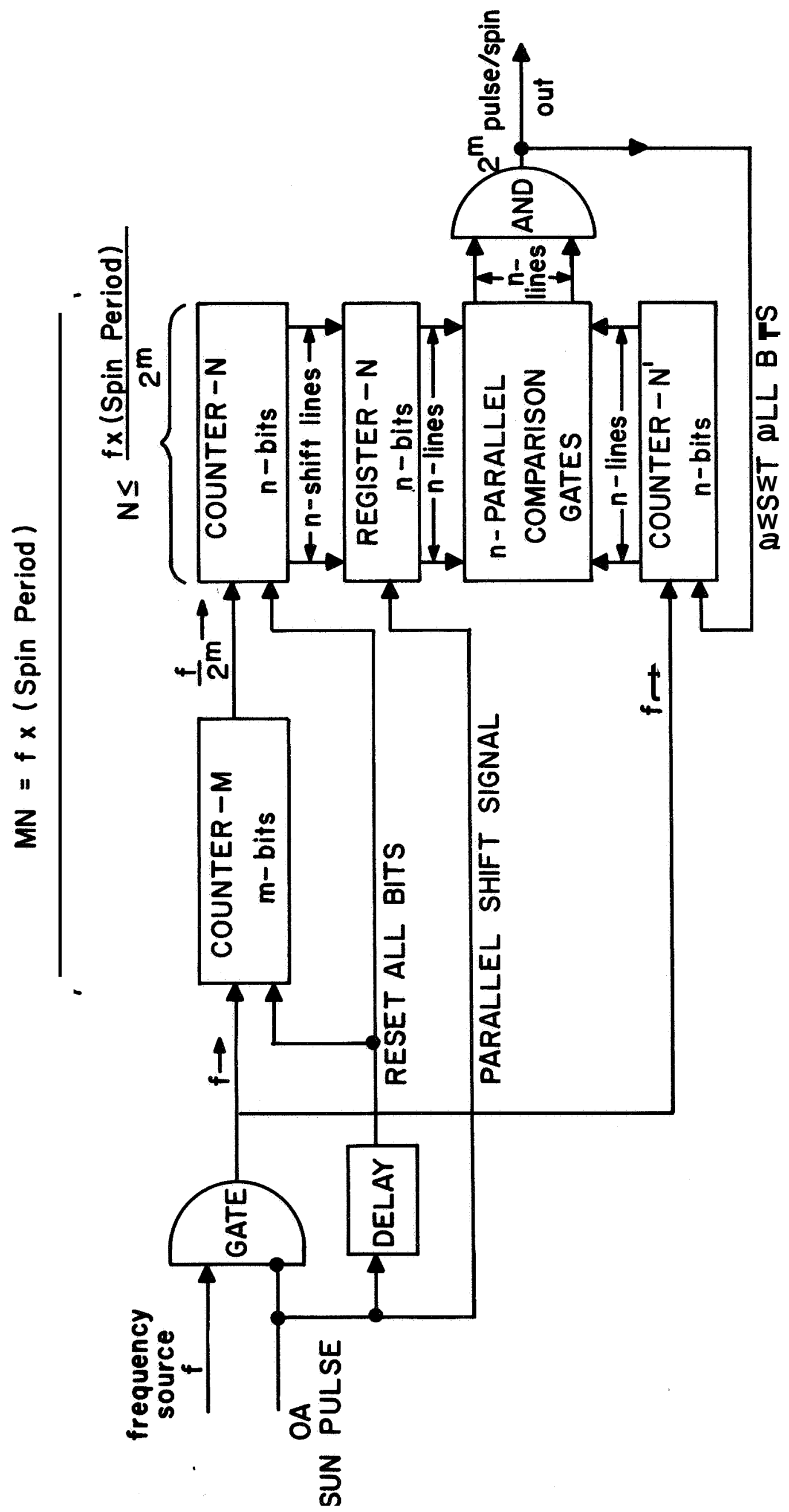

M
$\frac{\omega}{0}$
$\frac{0}{4}$ 


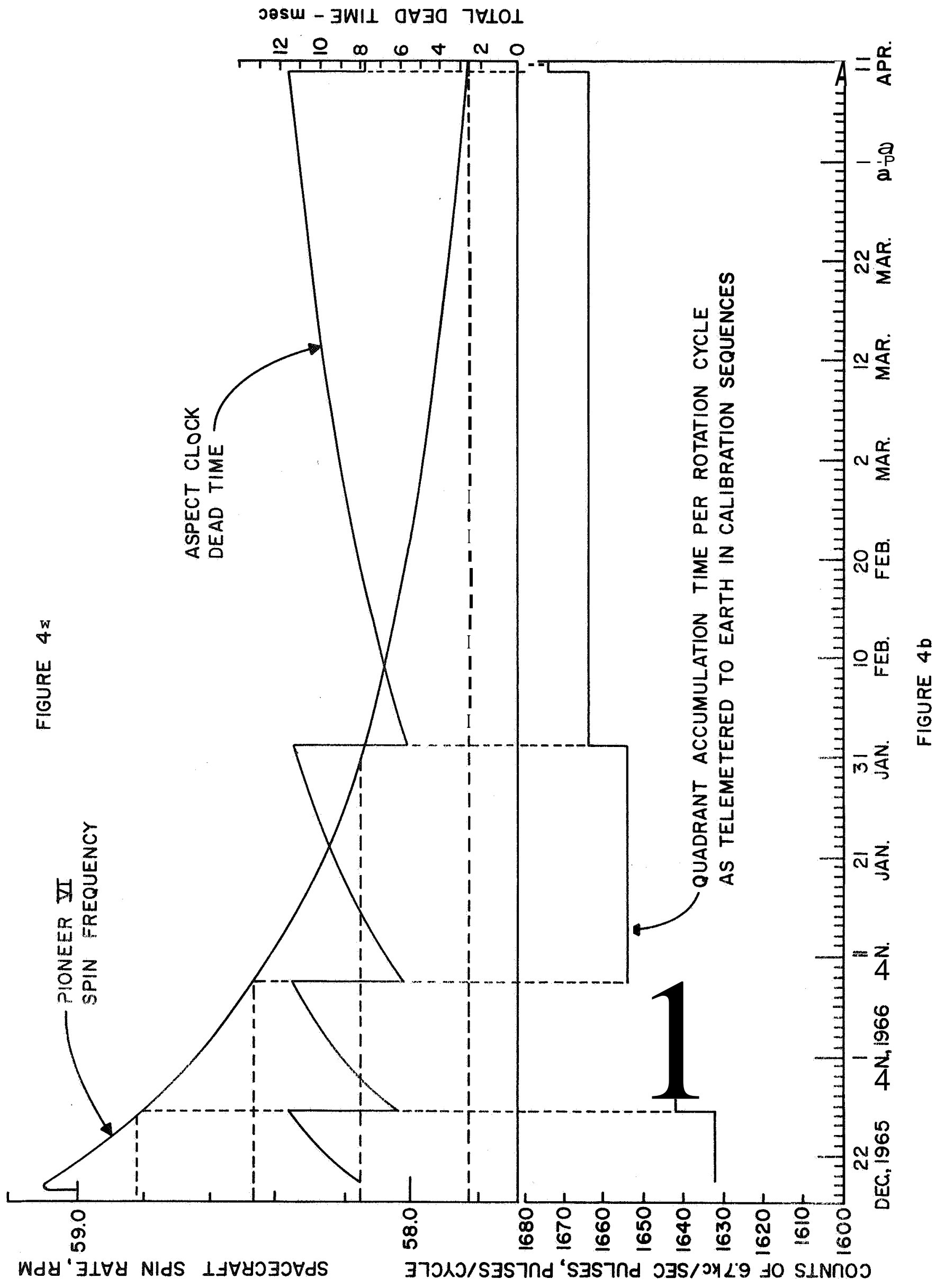




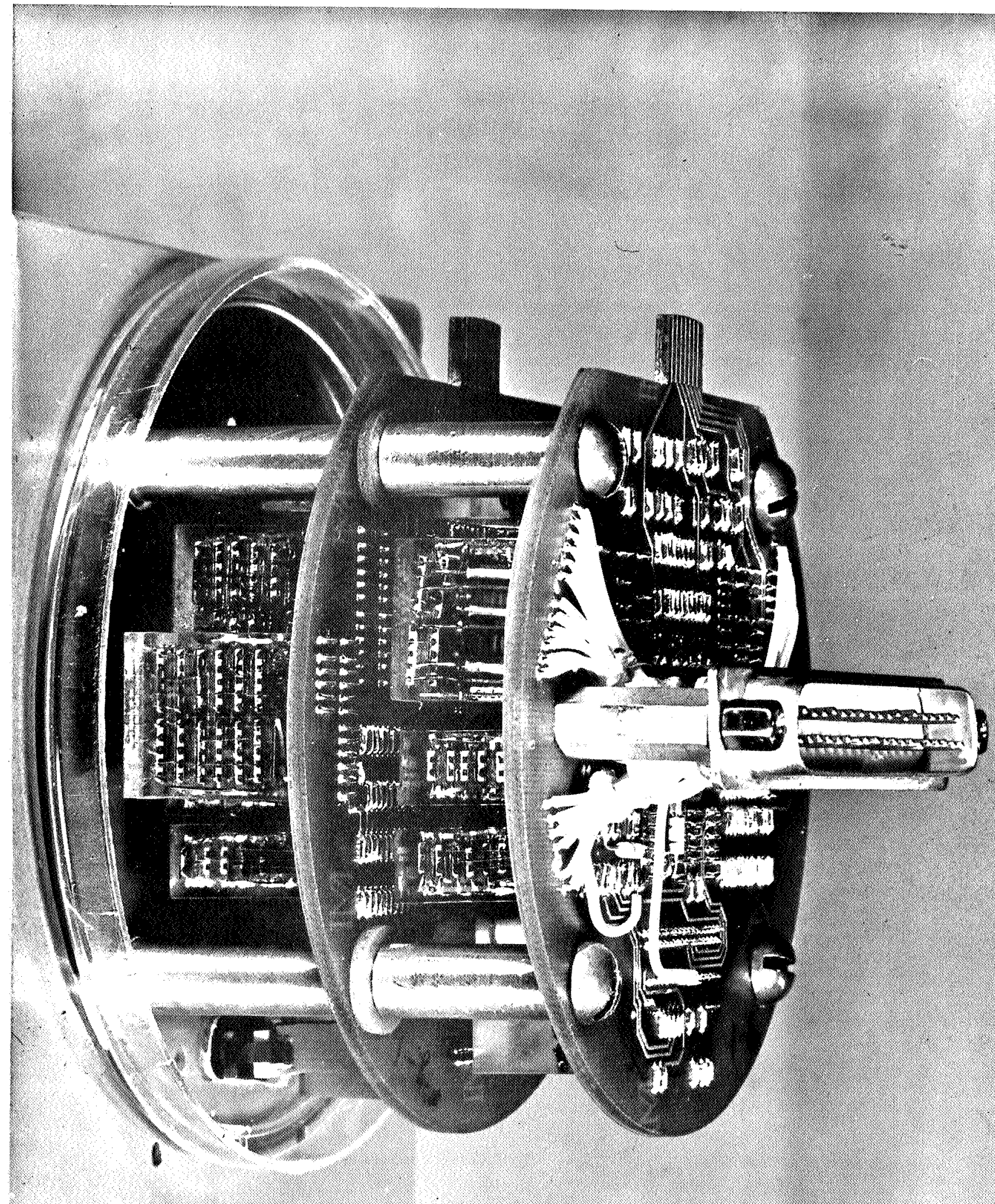

FIGURE 5: Photo shows complete signal conditioner package containing approximately 400 integrated circuits in welded modules mounted on two laminated sixlayer printed circuit boards 3.0 inches in diameter. The "Aspect clock" comprises approximately one-seventh of this package. 


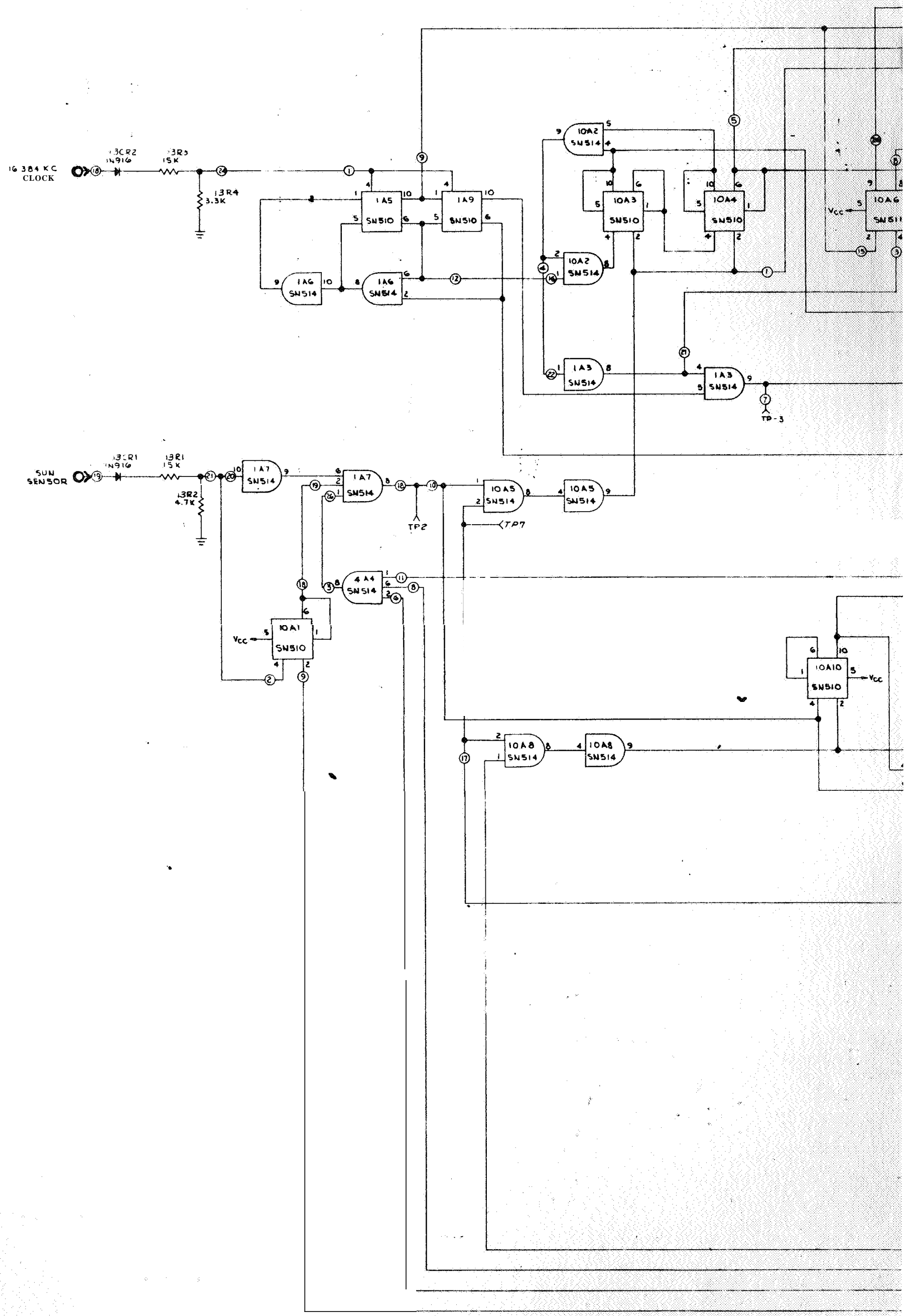

FIGURE 6: PIONEER ASPECT CLOCK SCHEMATIC 